\title{
A Multi Level Fuzzy Threshold Image Segmentation Method for Industrial Applications
}

\author{
P.Priyanka ${ }^{1}$, K.Vasudevarao ${ }^{2}$, Y.Sunitha ${ }^{3}$, Dr.B.Sridhar ${ }^{4}$ \\ ${ }^{1,2,3,4}$ Department of Electronics and Communication Engineering,Lendi institute of Engineering and Technology, \\ Vizianagaram,India.
}

\begin{abstract}
The non-contact measuring and segmentation method is of primary concern for heavy forging industrial applications. Such a measuring method, if successful, can improve the productivity and quality of manufactured items, reduce human labor, minimize material waste, and even further permit real-time control. This proposed method concerns with the utilization a fuzzy logic automatic analysis thermal images as of industrial products for defect detection. An original two stages algorithm is presented based on the feature analysis of the thermal images obtained from the inspected product. Each object in the image is analyzed using fuzzy logic techniques. The first stage takes an automatic decision whether the current object can be classified as a defect from the geometrical point of view and the second stage takes the final decision by using "logical" criteria that are dependent on the product at hand and its quality requirement, These results indicate that the proposed approach is more practical and convenient than current methods for real-time, on-site measurements.
\end{abstract}

\section{Introduction}

Image segmentation is one of the most important techniques in image processing. The most important challenge in this field is image vision. Dividing images into distinct regions that go through the recognition and understanding process is a crucial issue in image understanding. The division or splitting process is known as the segmentation process. The set of fuzzy threshold functions is defined to be a fuzzy set over the set of functions. All threshold functions have full memberships in this fuzzy set. Thus the distance measure between a non-linearly separable function and the set of all

threshold functions can be defined and investigated. It is widely used in different applications such as computer vision, digital pattern recognition, robot vision, etc.

ZhiweiYe et al[1].sum, a multi-level thresholding method based on GSO and Otsu is presented and the process is illustrated in the paper. Moreover, the proposed method are contrasted to some multi-level Otsu thresholding methods optimized by GA, PSO and DE. Testing results display that the proposed method is able to gain the optimal solution among these methods, which is more robust than some other methods involved in the paper. However, it is noted that as the dimension of threshold values increase, GSO is not entirely satisfactory.

Zhenhua Wang et al [2].In this paper, we propose a new image segmentation method which is based on the fast FCM clustering algorithm and multi-histogram (MHFFCM). MHFFCM can segment the image much more effectively than the conventional FCM clustering algorithm. In addition, the computation cost is much less than the conventional FCM because the number of the data set is decreased greatly. Experimental results validate that the method is a very effective method for image segmentation.

ASSAS Ouarda et al [3]. In this work, we propose an approach to find the optimal thresholds of an image, based on the concept of type-2 fuzzy c partition and the PSO algorithm. The main idea of this work was to introduce the use of type- 2 fuzzy sets into fuzzy thresholding. The experimental results have shown the effectiveness and usefulness of the proposed methods for image multi-level thresholding. Further research is needed to improve the proposed approach. Improvement of better membership function design it greatly contributes to getting better thresholding technique. Moreover, it is necessary to study the influence of linguistic hedges parameter to image with a certain characteristic. Other membership function formula can also be proposed and used.

Arti Taneja et al [4].In this paper, an overview of various image segmentation is presented. From the survey, it is found out that intensity and texture based methods based on level set function efficiently segment the image. The quality of the image with the presence of noise analyzed and improved on texture based methods. The analysis of the performance parameters such as accuracy, confusion matrix parameters, Kappa's coefficient on traditional methods showed the suitable methods were required for improvement. The effective refinement of the boundary on Optical Disk (OD) in retinal images performed by the level set methods.

Nita M. Nimbarte et al [5]. In this paper, we have analyzed and compared Otsu and MCVT thresholding algorithm for color image segmentation. For region merging, JND property of human visual perception has been employed. Experimental results demonstrate that Otsu method is superior to MCVT method. 
Gulpi Qorik Oktagalu Pratamasunu et al [6].In this paper, an automatic image thresholding method based on the index of fuzziness and fuzzy similarity measure is presented. This work overcomes some limitations of an existing method concerning the definition of the initial seed intervals and low contrast images. Index of fuzziness is used to find the center of the fuzzy region and initial seed intervals on the histogram. After defining the initial seeds, a similarity measurement process is started to find the threshold point by calculating a fuzzy similarity measure that considers fuzzy membership function and shape of the histogram. According to the analysis, we can conclude that the proposed method has better performance than the Lopes method and the Otsu thresholding. Evaluation of several test images with a different level of noises illustrates the robustness of the proposed method on images with noises.

Zhenhua Wang et al [7]. In this paper, we propose a new image segmentation method which is based on the fast FCM clustering algorithm and multi-histogram (MHFFCM).MHFFCM can segment the image much more effectively than the conventional FCM clustering algorithm. In addition, the computation cost is much less than the conventional FCM because the number of the data set is decreased greatly. Experimental results validate that the method is a very effective method for image segmentation.

\section{Multileve Fuzzyentropy}

A set $\mathrm{A}$ is defined as a collection of element which either belongs or does not belongs to set A. According to fuzzy set which is a generalization of set A, an element can partially belong to a set A and this set $\mathrm{A}$ is defined as

$A=\left\{\left(x, \mu_{A}(X)\right) \mid x \in X\right\}$

Where $0 \leq \mu_{A}(X) \leq 1$ and $\mu_{A}(X)$ is called the membership function, which measures the closeness of $\mathrm{x}$ to A. The trapezoidal membership function which is used in this paper is to estimate the membership of $\mathrm{n}$ segmented regions, $\mu_{1}, \mu_{2}, \ldots . \mu_{n}$ by using $2 \times(\mathrm{n}-1)$ unknown fuzzy parameters, namely $a_{1}, c_{1} \ldots . a_{n-1}, c_{n-1}$ where $0 \leq a_{1} \leq c_{1} \leq \ldots \leq a_{n-1} \leq c_{n-1} \leq L-1$

derived for $\mathrm{n}$ level thresholding

Then the following membership function can be

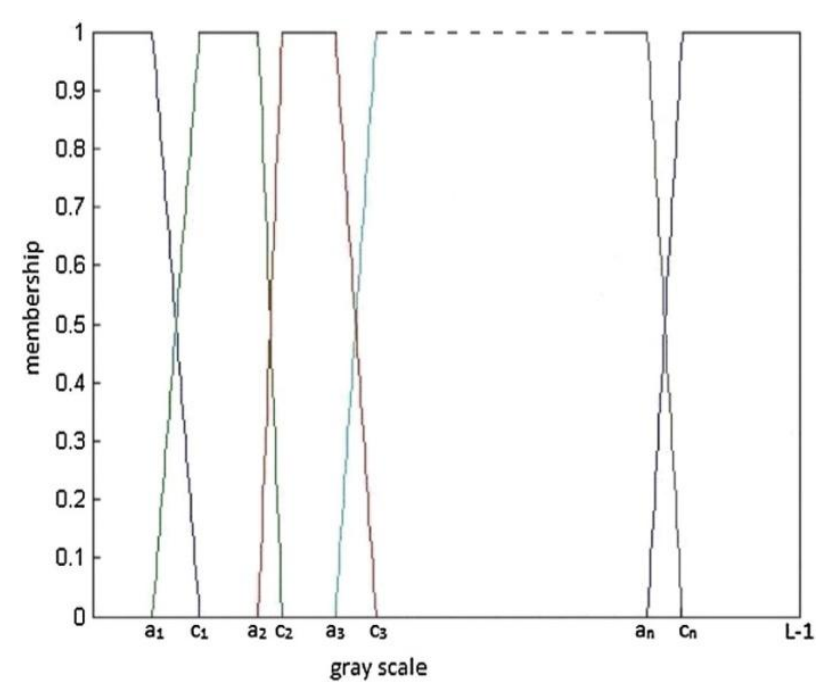

Fig. 1. Fuzzy membership function for $\mathrm{n}-$ level thresholding

$$
\begin{gathered}
\mu_{1}(k)=\left\{\begin{array}{cc}
1 & k \leq a_{1} \\
\frac{k-c_{1}}{a_{1}-c_{1}} & a_{1} \leq k \leq c_{1} \\
0 & k>c_{1}
\end{array}\right. \\
\mu_{n-1}(k)=\left\{\begin{array}{cl}
0 & k \leq a_{n-2} \\
\frac{k-a_{n-2}}{c_{n-2}-a_{n-2}} & a_{n-2}<k \leq c_{n-2} \\
\frac{k-c_{n-1}}{a_{n-1}-c_{n-1}} & a_{n-1}<k \leq a_{n-2} \\
0 & k>c_{n-1}
\end{array}\right.
\end{gathered}
$$


$\mu_{n}(k)=\left\{\begin{array}{c}1 k \leq a_{n-1} \\ \frac{k-a_{n-2}}{c_{n}-a_{n}} a_{n-1}<k \leq c_{n-1} \\ 1 k>c_{n-1}\end{array}\right.$

The maximum fuzzy entropy for each segment of $\mathrm{n}$-level segments can be defined by

$H_{1}=-\sum_{i=0}^{L-1} \frac{P_{i} * \mu_{1}(i)}{P_{1}} * \ln \left(\frac{P_{i^{*}} \mu_{i}(i)}{P_{1}}\right)$,

$H_{2}=-\sum_{i=0}^{L-1} \frac{P_{i} * \mu_{2}(i)}{P_{2}} * \ln \left(\frac{P_{i} * \mu_{2}(i)}{P_{2}}\right)$

$H_{n}=-\sum_{i=0}^{L-1} \frac{P^{*} * \mu_{n}(i)}{P_{n}} * \ln \left(\frac{P^{*} * \mu_{n}(i)}{P_{n}}\right)$

The maximum fuzzy entropy for each segment of $\mathrm{n}$-level segments can be defined by

Where,

$P_{1}=\sum_{i=0}^{L-1} P_{i} * \mu_{1}(i), P_{2}=\sum_{i=0}^{L-1} P_{i} * \quad \mu_{2}(i), \ldots, P_{n}=\sum_{i=0}^{L-1} P_{i} * \mu_{n}(i)$

The optimum value of parameters can be obtained by maximizing the total entropy

$\varphi\left(a_{1}, c_{1}, \ldots, a_{n-1}, c_{n-1}\right)=\operatorname{Argmax}\left(\left[H_{1}(t)+H_{2}(t)+\cdots+H_{n}(t)\right]\right)$

In order to optimize the eq efficiently and also to reduce the time complexity of the proposed method, a global optimization technique is needed. The (n-1) no of threshold values can obtain using the fuzzy parameters in the following way:

$$
\begin{gathered}
t_{1}=\frac{\left(a_{1}+c_{1}\right)}{2}, t_{2}=\frac{\left(a_{2}+c_{2}\right)}{2}, \ldots, \\
t_{n-1}=\frac{\left(a_{n-1}+c_{n-1}\right)}{2}
\end{gathered}
$$

\section{Differential Evolution}

Differential evolution which is population-based global optimization algorithm, was proposed by Storn in 1997.The $\mathrm{i}^{\text {th }}$ individual (parameter vector) of the population at generation (time) $\mathrm{t}$ is a $\mathrm{D}$-dimensional vector containing a set of D optimization parameters:

$\overrightarrow{Z_{l}}(t)=\left[Z_{i, 1}(t), Z_{i, 2}(t), \ldots, Z_{i, D}(t)\right]$

For changing the population members $\vec{Z}_{l}(t)$, in each generation, a donor vector $\vec{Y}_{l}(t)$ is created. This method is used for the creation of the donor vector that distinguishes the various DE schemes. In one of the earliest variants of DE now called DE/rand/1 scheme is used to create a donor vector $\vec{Y}_{l}(t)$ for every $\mathrm{i}^{\text {th }}$ member three other parameter vectors(say the $\mathrm{r} 1, \mathrm{r} 2$ and $\mathrm{r} 3$-th vectors such that $r_{1}, r_{2}, r_{3} \in[1, N P]$ and $r_{1} \neq r_{2} \neq r_{3}$ ) are chosen at random from the current population. The donor vector $\vec{Y}_{l}(t)$ is obtained multiplying a scalar number $\mathrm{F}$ with the difference of any two of the three. The process for the jth component of the ith vector may be expressed as,

$\overrightarrow{Y_{l, j}}(t)=Z_{r_{1}, j}(t)+F \cdot\left(Z_{r_{2}, j}(t)-Z_{r_{2}, j}(t)\right.$

A 'binomial' crossover operation takes place to increase the potential diversity of the population. This is performed on each of the $\mathrm{D}$ variables whenever the selected number between 0 and 1 is within the $\mathrm{Cr}$ value. In this case, the number of parameters inherited from the mutant has a (nearly) binomial distribution. Thus for each target vector $\overrightarrow{Z_{l}}(t)$ a trial vector $\overrightarrow{R_{l}}(t)$ is created in the following fashion:

$$
\begin{aligned}
& R_{i, j}(t)=Y_{i, j}(t) \text { if rand } \text { rand }_{j}(0,1) \leq c_{r} \text { or } j=m(i) \\
& \quad=Z_{i, j}(t) \text { otherwise }
\end{aligned}
$$


For $\mathrm{j}=1,2 \ldots \mathrm{D}$ and $\operatorname{rand}_{j}(0,1) \in[0,1]$ is the $\mathrm{j}^{\text {th }}$ evaluation of a uniform random number generator. $m(i) \in[1,2, \ldots, D]$ is a randomly chosen index to ensure that $\overrightarrow{R_{l}}(t)$ gets at least one component from $\overrightarrow{Z_{l}}(t)$. Finally 'selection' is performed in order to determine which one between the target vector and trial vector will survive in the next generation i.e. at time

$t=t+1$. If the trial vector yields a better value of the fitness function, it replaces its target vector in the next generation; otherwise, the parent is retained in the population:

$$
\begin{aligned}
\qquad \overrightarrow{Z_{l}}(t+1)=\overrightarrow{R_{1}}(t) \text { if } f\left(\overrightarrow{R_{1}}(t)\right)>f\left(\overrightarrow{Z_{l}}(t)\right) \\
=\overrightarrow{Z_{l}}(t) \text { if } f\left(\overrightarrow{R_{1}}(t)\right) \leq f\left(\overrightarrow{Z_{1}}(t)\right) \text { (9) }
\end{aligned}
$$

Where $\mathrm{f}($.$) is the function to be maximized$

\subsection{Membership Functions:}

The mutation strategy and the control parameters, namely scaling factor $(F)$, crossover rate $(C R)$, and population size $(N P)$, play the major roles in the success of a DE. Choosing the appropriate mutation operator and parameter values for a particular problem is a difficult task because it is a problem dependent, timeconsuming, and trial-and-error process. We may need mutation strategy and parameter values at different stages during the evolution process. In the beginning of the evolution in order to search larger regions in the space, we need a higher degree of exploration than exploitation. We choose the mutation operator that possesses high exploration ability and the control parameter that promote the diversity. The mutation operator which favors local search can be chosen along with the control parameters that emphasize the exploitation. If we know the state of the evolution process, we may decide whether we should emphasize on exploration or exploitation, and choose suitable parameter values or the mutation strategies.

There is a unique possible method that we can observe the status of the total process is utilizing the performance metrics. Most of the performance metrics are calculated at the end of the development in order to determine the quality of the obtained non dominated front.For evidence, The true Pareto front system is used to find generational distance. We cannot find the true Pareto front is available during the evolution search. By using Three properties of non dominated front we can measure the quality of population, namely, the convergence, uniform distribution, and extensiveness.Hence we have very less chances to measure the convergence, uniform distribution, and extensiveness of the population. Hence, we achieved three performance metrics to measure the three properties of the obtained non dominated solutions. The proposed fuzzy-based multiobjective differential evolution (FMDE) employs hyper volume, spacing, and maximum distribution as the input to the fuzzy inference rules that adaptively adjust the widget parameters for the mutation scheme which is the diversity factor and the greedy factor every generation in order to maintain balance between the exploration and exploitation abilities of the population during the search process. Moreover, the advanced version of FMDE (AFMDE) is introduced. AFMDE mix the adaptive $C R$ by fuzzy rules.

\subsubsection{Differential Mutation:}

The differential mutation is the key to success, when K. Price invented Genetic Annealing and soon after that R. Storn cooperated with him in order to solve the Tchebychev polynomial fitting problem by genetic annealing. In continuous space the above problem is formulated, that's why it changed from bit-string to floating-point encoding, and from logical operators to arithmetic ones. Although, they were doing some experiments, they discovered the differential mutation. They also observed that using the composition of differential mutation, discrete recombination, and pair-wise selection, the annealing mechanism is no longer needed. They removed the annealing factor and then DE was born.

\subsubsection{Differential Evolution (DE) \\ Fundamentals :}

The original version of DE is described in this section. A flowchart of the classical DE algorithm is shown in Figure. Like other evolutionary algorithms, it starts with the randomly initializing a population in the search space. In DE community, an individual of the population is called a parameter vector; NP parameter vectors form a population. Then the population enters the evolution loop: mutation, crossover, and selection operations. These three operations will be repeated until the stopping criterion is met.

\section{The population}

The DE population represents NP D-dimensional parameter vectors. These vectors are real numbers. The current population is

$$
\begin{aligned}
& P_{x, G} \text { composed of vectors } X_{i, G} \text { as } \\
& \qquad P_{x, G}=\left\{x_{i, G}\right\}, i=1,2, \ldots, N P, G=1,2, \ldots, g_{\max }
\end{aligned}
$$


$X_{i, G}=\left\{X_{j, i, G}\right\}, j=1,2, \ldots, D$

where $N P$ is the number of population vectors, $G$ is the generation number, and $D$ is the dimensionality of the vector.

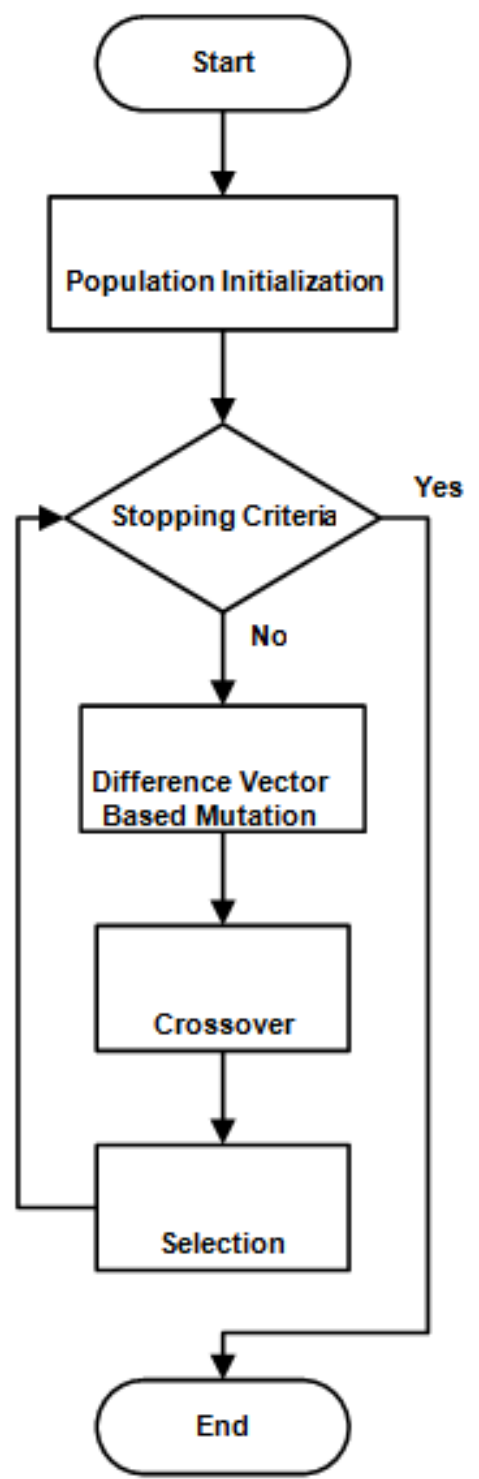

Figure 2 : The classical DE flowchart.

2. The population initialization :

The population is initialized by

$$
X_{i, j}=\operatorname{rand}_{j}[0,1) \cdot\left(b_{j, U}-b_{j, L}\right)+b_{j, L}
$$

where $b_{\mathrm{L}}$ and $\mathrm{b}_{\mathrm{U}}$ is the lower and upper bounds of the vectors $\mathrm{X}_{\mathrm{i}, \mathrm{j}}$ at generation $G=0,[0,1)$ is uniformly distributed random number within the range $[0,1)$. This number is generated for each element $j$ of vectors.

\section{Mutation :}

After population initialization, $\mathrm{DE}$ uses the mutation to generate a candidate vector called the mutant vector $\mathrm{V}_{\mathrm{i}, \mathrm{G}}$ with respect to the target vector $\mathrm{X}_{\mathrm{i}, \mathrm{G}}$ by adding the base vector to weighted difference vectors. This key operation of DE is shown

$\mathrm{DE} / \mathrm{rand} / 1: V_{i, G}=x_{r_{1}, G}+F\left(X_{r_{2}, G}-X_{r_{3}, G}\right)$ 
The random integers $i, r 1, r 2$ and $r 3$ are randomly generated and mutually exclusive within the range $[1, N P]$, used as indices to index the current parent vectors. As can be seen from, we need at least three vectors in a population. $F$ is a positive scaling factor, which manages the trade-off between exploration and exploitation. Originally, $F \in(0,1+)$ means while there is no upper limit on $F$, effective values are seldom greater than 1.0 . Storn and Price suggested that $F$ should be 0.8 for general problems. The original mutation strategy is called $\mathrm{DE} / \mathrm{rand} / 1$ as $\mathrm{DE} / \mathrm{x} / \mathrm{y} / \mathrm{z}$ represents DE/base vector/number of difference vectors/crossover. There are two types of crossover operators, namely, binomial and exponential crossovers. Therefore, DE/rand/1/bin is the DE/rand/1 mutation scheme with the binomial crossover and DE/rand/1/exp is the DE/rand/1 mutation scheme with the exponential crossover. A pictorial example of DE/rand/1 is illustrated in Figure

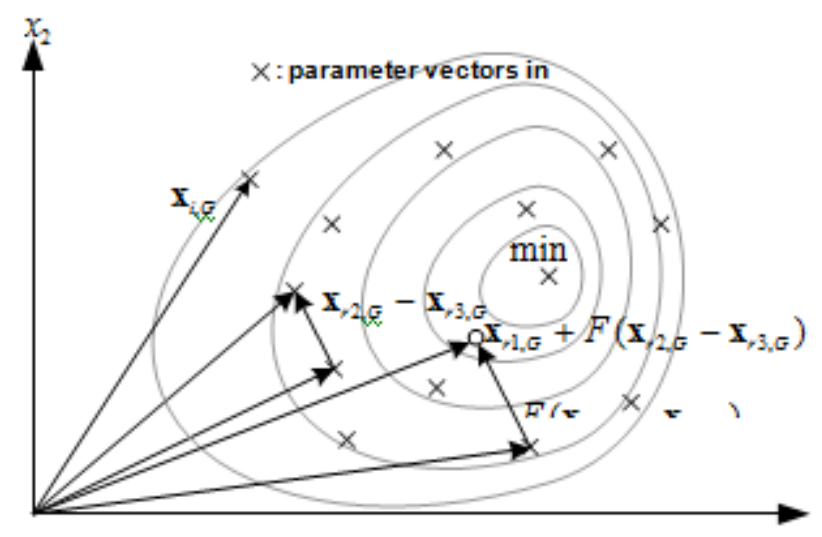

Figure 3 : Illustration of DE/rand/1 scheme in 2-D space.

There are many mutation strategy proposed by many researchers. The most commonly used mutation operations are ,

$\begin{array}{rr}\text { DE/rand/2: } V_{i, G}=X_{r_{1}, G}+F\left(X_{r_{2}, G}-X_{r_{3}, G}+\right. & F\left(X_{r_{4}, G}-X_{r_{5}, G}\right) \\ \text { DE/best/1: } V_{i, G}=X_{\text {best }, G}+F\left(X_{r_{1}, G}-X_{r_{2}, G}\right)(13 \mathrm{~b}) & \\ \begin{array}{rr}\text { DE/best/2: } v_{i, G}=X_{\text {best }, G}+F\left(X_{r_{1}, G}-X_{r_{2}, G}\right)+ \\ \text { DE/current-to-best/2: } \boldsymbol{\varpi}_{l, \Gamma}=\xi_{l, \Gamma}+\Phi\left(\xi_{\left.\beta \varepsilon \sigma \tau, \Gamma-\xi_{l, \Gamma}\right)}\right.\end{array} & F\left(X_{r_{3}, G}-X_{r_{4}, G}\right) \\ & +\Phi\left(\xi_{\left.\rho 1, \Gamma-\xi_{\rho 2, \Gamma}\right)(13 \delta)}\right. \\ \text { DE/rand-to-best/2: } V_{i, G}= & X_{i, G}+F\left(X_{\text {best }, G}-X_{r_{2}, G}\right)+\end{array}$

Trigonometric mutation :

$V_{i, G}=\frac{X_{r_{1}, G}+X_{r_{2, G}}+X_{r_{3}, G}}{3}+\left(P_{2}-P_{1}\right)\left(X_{r_{1}, G}-X_{r_{2}, G}\right)+\left(P_{3}-P_{2}\right)\left(X_{r_{2}, G}-X_{r_{3}, G}\right)+\left(P_{1}-P_{3}\right)\left(X_{r_{3}, G}-X_{r_{1}, G}\right)$

Where

$P_{1}=\frac{\left|f\left(X_{r_{1}, G}\right)\right|}{P^{\prime}}, P_{1}=\frac{\left|f\left(X_{r_{2}, G}\right)\right|}{P^{\prime}}, P^{\prime}=\left|f\left(X_{r_{1}, G}\right)\right|+\left|f\left(X_{r_{2}, G}\right)\right|+\left|f\left(X_{r_{3}, G}\right)\right|$,

and $f(\mathbf{x})$ is the objective function. The indices $r 1, r 2, \ldots, r 5$ are randomly generated integers within the range $[1, N P]$ and they are mutually exclusive and different from $i$. For each mutant vector, we must generate new indices. $\mathrm{X}_{\text {best }, \mathrm{G}}$ is the individual vector that provides the best objective value in the population in the generation $G$.

\section{Crossover:}

After we get the mutant vectors from the mutation operation, we perform the crossover operation in order to increase the potential diversity of the population. Crossover is applied to each pair of the target vector and it's mutant vector then we obtain a trial vector. The DE family algorithms can use two types of crossover schemes: the binomial (uniform) crossover and the exponential crossover.

\subsection{Binomial crossover}

The binomial crossover is the discrete recombination operator by:

$U_{i, G}=\left\{u_{j, i, G}\right\} \quad=\left\{\begin{array}{lr}v_{j, i, G} \text { if } \text { rand }_{j}(0,1) \leq C R \text { or } j=j_{\text {rand }} \\ x_{j, i, G}\end{array}\right.$ 
The crossover rate or so called crossover probability $C R \in[0,1]$ is a user-defined constant that controls the fraction of parameter values copied from the mutant vector. $C R$ is suggested to be $0.8-1$. Here the crossover operator is uniform in the sense that each parameter of the mutant vector, regardless of its location has the same probability, $C R$, of inheriting its value from a given to the trial vector. For this reason, uniform crossover does not exhibit a representation bias. A pictorial example for binomial crossover is illustrated by Figure 2.3. The target vector $\mathrm{X}_{\mathrm{i}, \mathrm{G}}$ exchanges its parameters with the mutant vector $\mathrm{V}_{\mathrm{i}, \mathrm{G}}$ to in order to form the trial vector $\mathrm{u}_{\mathrm{i}, \mathrm{G}}$.

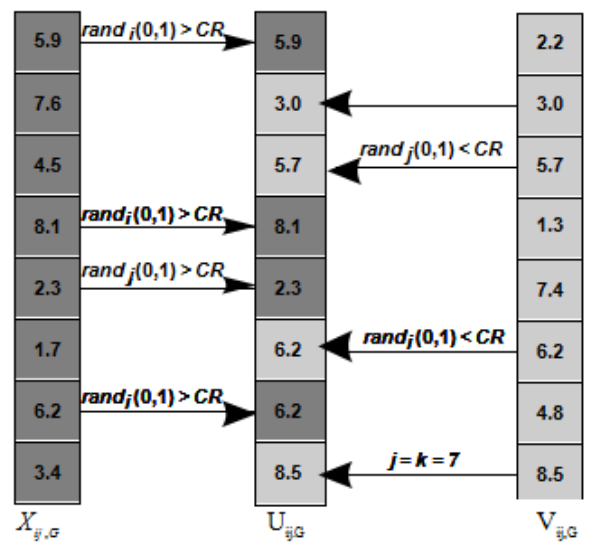

Figure 4 : A pictorial example for binomial crossover.

\subsection{Exponential crossover}

DE exponential crossover is functionally equivalent to two-point crossover in GA. In exponential crossover, we begins with randomly choosing an integer $n$ from the interval $[1, D]$ as a starting point in the target vector, from where the exchange of parameters with the mutant vector starts. Another integer $L$ is also randomly chosen from the range $[1, D]$.

After choosing $n$ and $L$ the trial vector is generated by

$U_{i, G}=\left\{u_{j, i, G}\right\}$

$U_{j, i, G}=\left\{\begin{array}{c}v_{j, i, G} j=\langle n\rangle_{D},\langle n+1\rangle_{D}, \ldots,\langle n+L-1\rangle_{D} \\ x_{j, i, G} \text { all other } j \in[1, D]\end{array}\right.$

where \langle\rangle$_{D}$ denote a modulo function with modulus $D$.

The parameters of the trial vector $\mathrm{u}_{\mathrm{i}, \mathrm{G}}$ are inherited from the corresponding mutant vector $\mathrm{V}_{\mathrm{i}, \mathrm{G}}$ starting from index $n$ till the first time that $\operatorname{rand}(0,1)>C R$. All the remaining parameters of the trial vector are inherited from the corresponding target vector. An example of exponential crossover is shown in Figure 2.4. In this example, exponential crossover starts with $n=3$ which is randomly chosen and copied the parameter of the mutant vector to the trial vector until the first occurrence of $\operatorname{rand}(0,1)>C R$, the rest of parameters are copied from the target vector to the trial vector.

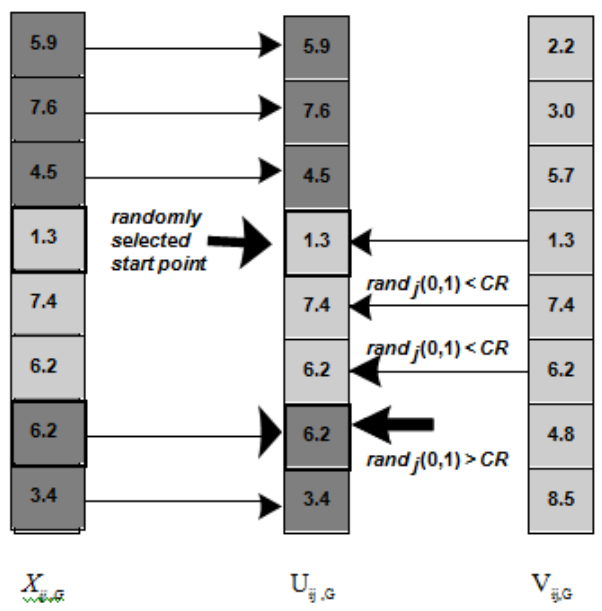

Figure 5 : A pictorial example of exponential crossover. 


\section{Flowcontrol For The Proposed Method:}

The proposed multilevel fuzzy thresholding based on the differential evolution in indentifying the defects or the faults in the systems at the industrial level can be done in different steps as following:

Step1: A threshold image of the system is takenas an input. The threshold values are fixed and it should be between 1 and the difference between the maximum and minimum gray level present in the image. The image is converted into gray scale image if it is in RGB.

Step 2: In order to get the thresholding image first we have to apply the histogram on the input image and next the differential evolution is applied on the image.

Step 3: After different membership functions applied on the histogram image the output after differential evolution is an fuzzy parameter vector.

Step 4 : The fuzzy parameter vector is obtained by performing the fuzzy entropy on the histogram image.

Step 5 : After applying the differential evolution technique an image having three threshold values and varying in three different segments is been obtained.

Step 6 : For the thersholded image first we have to detect the edges by the edge detection method. Like sobel operator and the cannon detection techniques.

Step 7: The weighted array is been calculated by the functions used in the matlab inorder to calculate the weigth array of any image.

Step 8 : The output of the original image having three different segments which are represented in three different colors varying three different temperature is been obtained.

The flow control of proposed methodology is as follows:

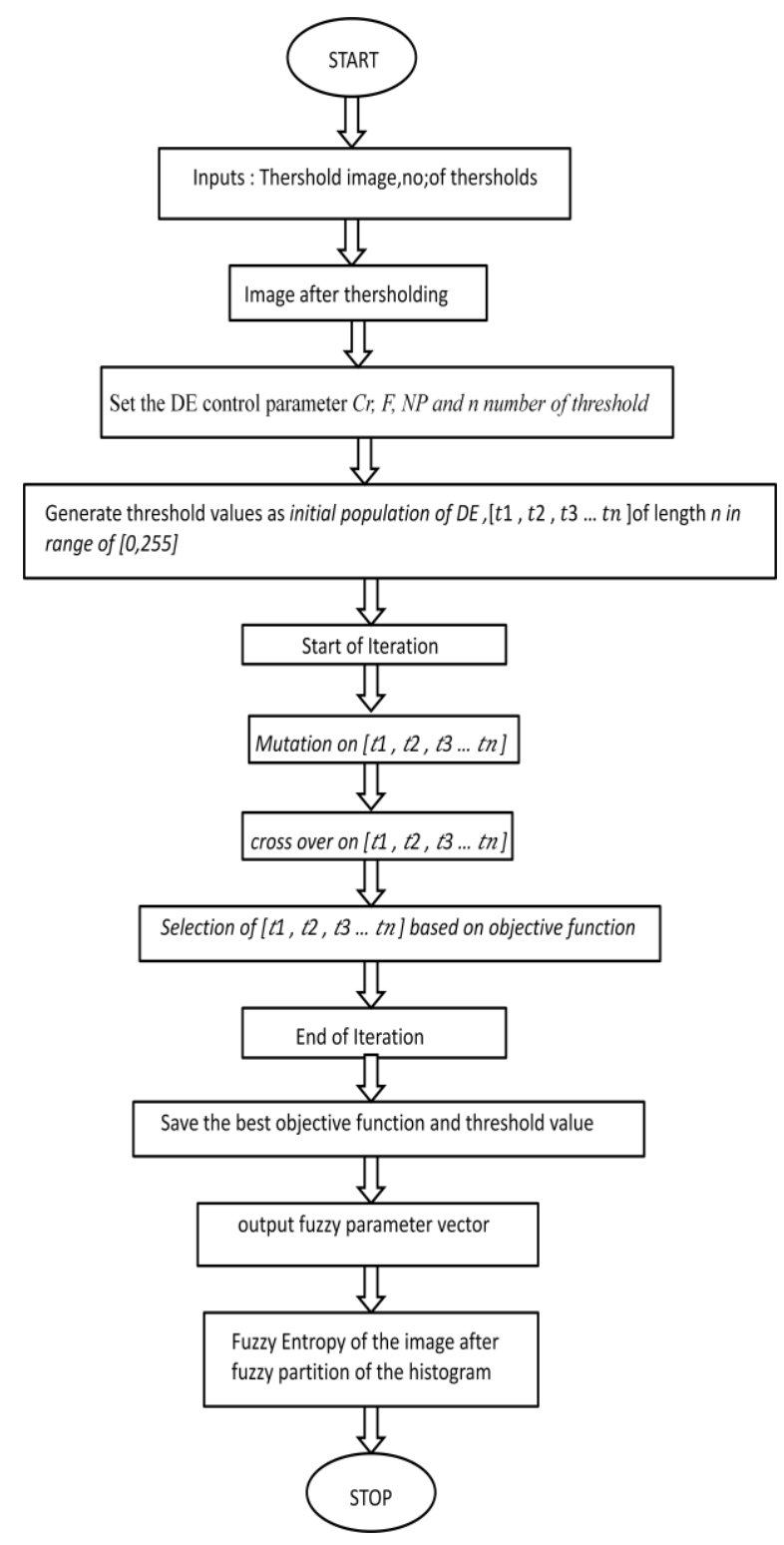




\section{Experimental Results}

The original thershold images that we are taken inorder to test or to detect the effects by using multithersholding are shown below:

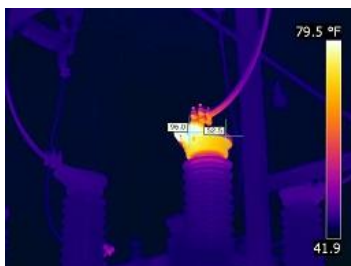

Fig:1

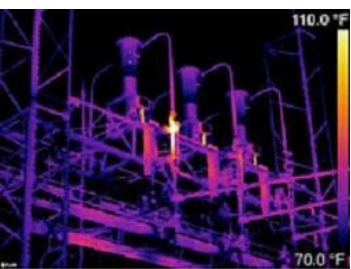

Fig:3

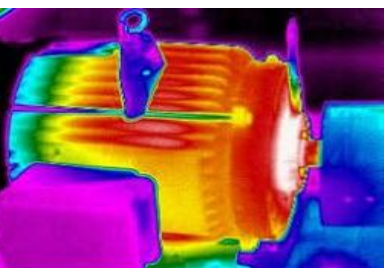

Fig:2

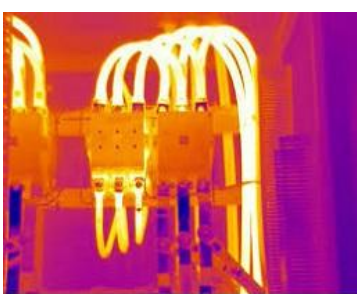

Fig:5

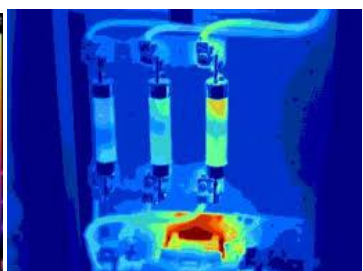

Fig:4

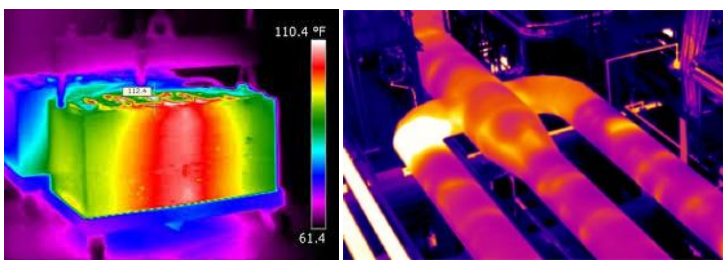

Fig:7

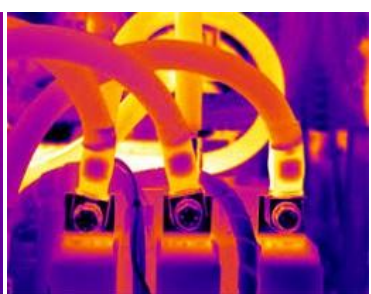

Fig:6

Fig:8

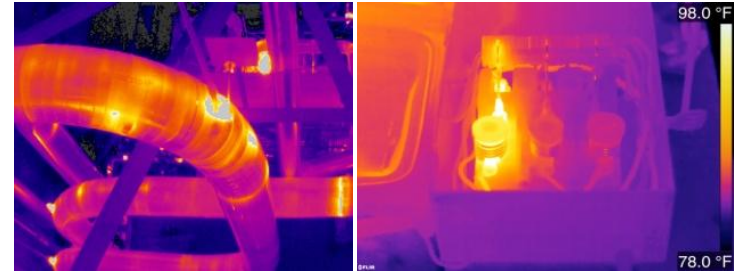

Fig:9

Fig:10

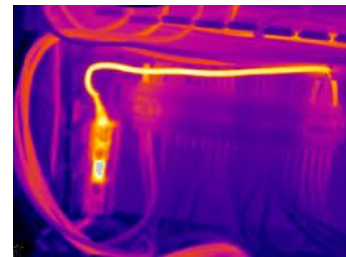

Fig:11

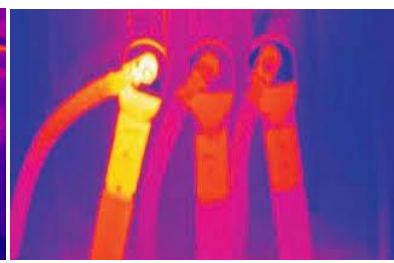

Fig:12

Following are the results that are illustrated by applying fuzzy multithresholding. Here the original color image is also been converted into a grayscale image. Here are a few stages that our output is been generated as follows 


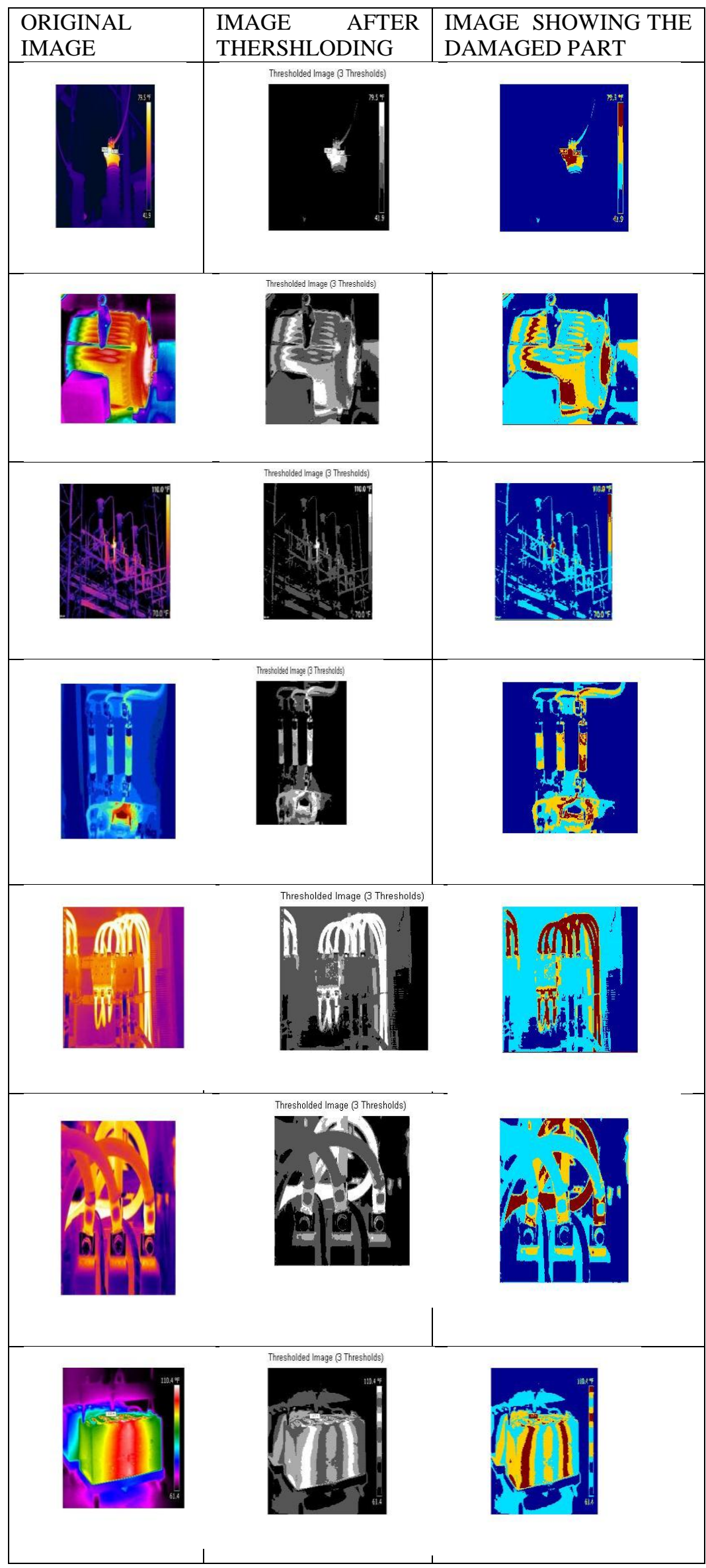




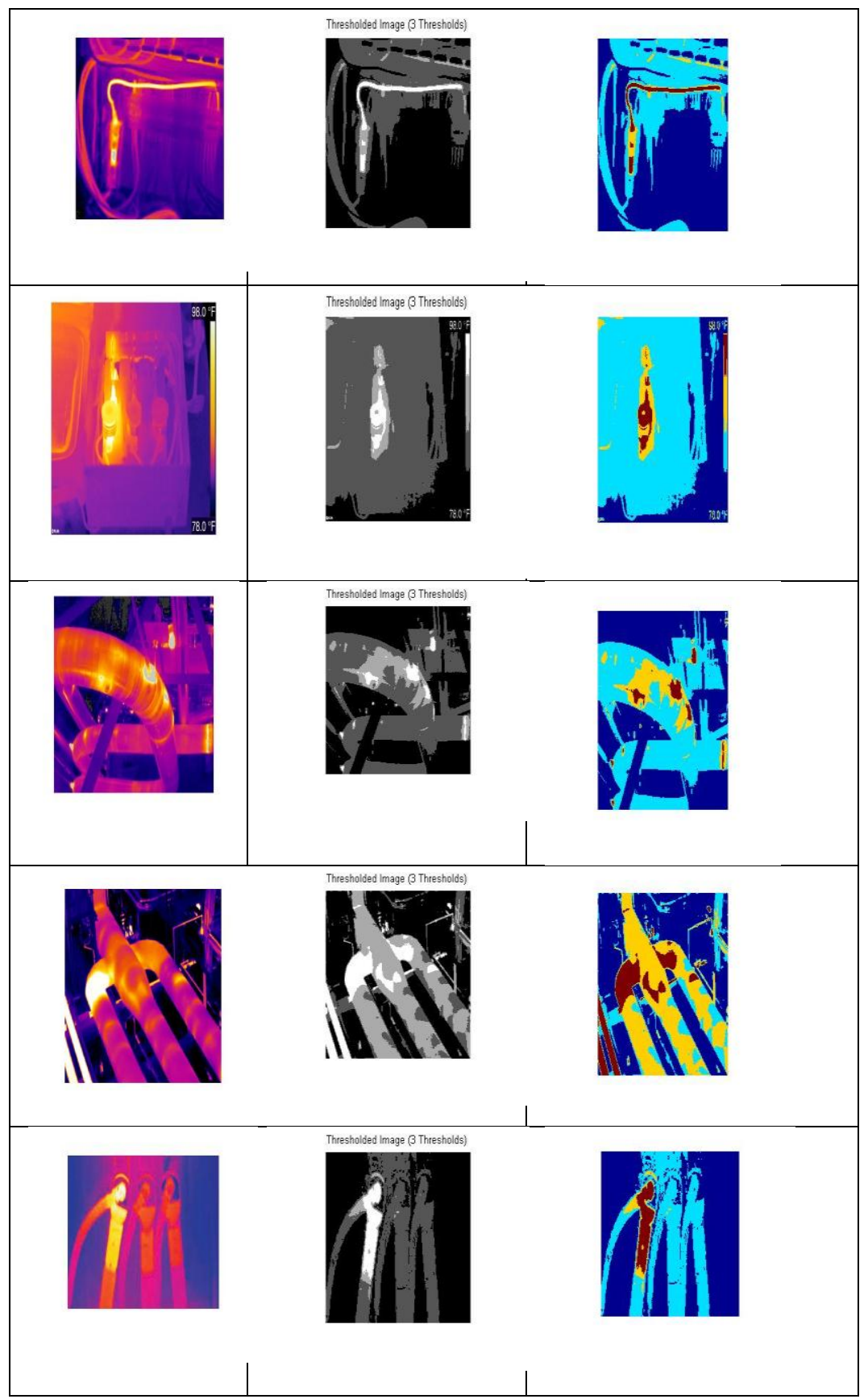

From the above figures, we can clearly observe the three levels of the threshold viewed in three different colors distinguished based on the severity of the fault in the system. Here in this, the red color represents the highly damaged area that is to be considered as a primary. Next color yellow represents the moderately damaged area. The blue represents the least damaged area that is to be considered. Here we considered only three colors as we done only three level thresholding so that only three segments are obtained. These three segments are been represented in three different colors.

\section{Conclusion}

In this paper, the discussion about the Multilevel Fuzzy threshold for image segmentation based on the differential evolution performs significantly better than OTSU based approaches. In this, we get the satisfactory results in comparison of visual statistics. Here by the usage of the three threshold values, we can differiante the fault regions in three levels. The three areas are represented in three different color densities. For achieving this several membership functions in the DE are used for the better separation of the segmented regions. At last, it is concluded that this technique adds speed and accuracy and could be implemented in order to get improved outcomes. 


\section{References}

[1]. Zhiwei Ye, "A Multi-level Thresholding Approach Based on Group Search Optimization Algorithm and Otsu". 2015 8th International Symposium on Computational Intelligence and Design.

[2]. Zhenhua Wang, “A New Multi-threshold Segmentation Method Based on MHFFCM”. Proceedings of the 2006 IEEE International Conference on Information Acquisition August 20 - 23, 2006, Weihai, Shandong, China.

[3]. ASSAS Ouarda *,'Image Thresholding Using Type-2 Fuzzy C-Partition Entropy and Particle Swarm Optimization Algorithm". Laboratory Analysis of Signals and Systems (LASS) University of M'sila, M'sila, Algeria

[4]. Arti Taneja, “A Performance Study of Image Segmentation Techniques". 978-1-4673-7231-2/15/\$31.00 @2015 IEEE.

[5]. Nita M. Nimbarte," Multi-level Thresholding Algorithm For Color Image Segmentation". 2010 Second International Conference on Computer Engineering and Applications.

[6]. Gulpi Qorik Oktagalu Pratamasunu," Image Thresholding Based on Index of Fuzziness and Fuzzy Similarity Measure”. 2015 IEEE 8th International Workshop on Computational Intelligence and Applications November 6-7, 2015, Hiroshima, Japan.

[7]. Zhenhua Wang," A New Multi-threshold Segmentation Method Based on MHFFCM". Proceedings of the 2006 IEEE International Conference on Information Acquisition August 20 - 23, 2006, Weihai, Shandong, China. 